\title{
Las memorias corporativas de Atresmedia y RTVE en la red: juegos malabares para la credibilidad
}

\author{
Isabel Arquero Blanco ${ }^{1}$ \\ Miguel Ángel OrTIz SOBRINO² \\ Universidad Complutense de Madrid
}

Recibido: 22/10/2014

Aceptado: 30/01/2015

\begin{abstract}
Resumen
En este artículo se comparan las memorias de responsabilidad social que la Corporación Radiotelevisión Española (RTVE) y Atresmedia, Corporación de Medios de Comunicación, S.A., difunden a través de sus webs corporativas. El análisis de estos informes, referentes al ejercicio 2012, permite revisar la información que los dos grupos de comunicación proyectan en la opinión pública, su transparencia y el diálogo que establecen con sus grupos de interés. Los dos grupos de comunicación omiten información sobre riesgos, propician la ambigüedad y mantienen un diálogo incompleto con sus grupos de interés.
\end{abstract}

Palabras clave: responsabilidad social, transparencia, informe anual, Atresmedia, RTVE.

\section{Corporate reports of Atresmedia and RTVE in the Web: Juggling for credibility}

\begin{abstract}
This article compares the 2012 reports on corporate social responsibility of Corporación Radiotelevisión Española (RTVE) and Atresmedia, Corporación de Medios de Comunicación, S.A., hosted on their corporate websites. The analysis of these reports allows to review the information that both communication groups project in public opinion, their transparency and dialogue with the stakeholders. The two compared reports overlook information on risks, foster ambiguity and incomplete dialogue with theirs stakeholders. Keywords: social responsibility, clarity, annual reporting, Atresmedia, RTVE.

\footnotetext{
${ }^{1}$ Profesora Asociada del Departamento de Comunicación Audiovisual y Publicidad 1 de la Facultad de Ciencias de la Información de la Universidad Complutense de Madrid (iarquero@ ucm.es).

${ }^{2}$ Profesor Contratado Doctor del Departamento de Periodismo IV (Empresa Informativa) de la Facultad de Ciencias de la Información de Madrid (maortiz@ccinf.ucm.es).
} 


\section{Introducción}

La responsabilidad social de las empresas (RSE) está vinculada a las consecuencias que las actuaciones empresariales tienen en la sociedad en general y en determinados grupos en particular. Puesto que las decisiones empresariales provocan impactos en los distintos colectivos implicados en su actividad, se les demanda un modelo de actuación responsable: «hacer suyas, de manera voluntaria, las preocupaciones sociales a través del diálogo transparente con sus grupos de interés $\rangle^{3}$. Los grupos de interés, también llamados stakeholders, están conformados por un conjunto amplio y diverso de personas o entidades: accionistas, competidores, consumidores, directivos, empleados, instituciones públicas, inversores, medios de comunicación, propietarios, proveedores y sindicatos, entre otros.

La RSE ha ido adquiriendo relevancia como resultado de los procesos de globalización de la economía y de la difusión mundial de la información propiciada por el desarrollo de las tecnologías de la información y la comunicación. Las preocupaciones y los contenidos que se han ido incorporando al corpus conceptual de la RSE han registrado una evolución paralela a la de los movimientos críticos y cívicos que han conformado el conjunto de la opinión pública en la segunda mitad del siglo XX.

Siguiendo a García Perdiguero, la corrupción y los escándalos financieros centraron la atención pública en los años setenta, mientras que en los años ochenta el interés se desplazó hacia los derechos de los consumidores, las prácticas comerciales fraudulentas y la denuncia de productos de consumo poco saludables como el alcohol y el tabaco. En la segunda mitad de los años ochenta, el debate público dominante se sitúa en las campañas de denuncia de las inversiones empresariales en países que no respetan los derechos humanos. A finales de los años noventa, los efectos negativos de la globalización y los procesos de reestructuración económica incrementan la crisis del bienestar y van agregándose a la definición de responsabilidad social de las empresas como principios a reprobar. Los problemas de exclusión económica y social «son la manifestación más concluyente de los límites de la racionalidad puramente económica en el mundo de hoy, ya que expresan de manera directa la terrible paradoja del crecimiento simultáneo de la riqueza y de la máxima pobreza» ${ }^{4}$.

Los principios que inspiran la RSE tienen como telón de fondo un concepto mucho más amplio que es el del desarrollo sostenible. Desde su formalización conceptual en 1987, en el conocido Informe Brundtland presentado a la Asamblea General de las Naciones Unidas por la Comisión Mundial sobre Medio Ambiente y Desarrollo, hasta nuestros días el desarrollo sostenible ha sido y continúa siendo una de las mayores preocupaciones de la comunidad internacional. Las conferencias Río 92 (1992), Río+10 (2002) y Río+20 (2012) constituyen claros ejemplos del renovado compromiso del sistema multilateral con el progreso humano y el bienestar global.

\footnotetext{
${ }^{3}$ BArRedA, J. (2006): «Propuestas del Foro de Expertos en RSE», Revista APD: Asociación para el Progreso de la Dirección, 212, 29.

${ }^{4}$ García Perdiguero, T. (2003): La responsabilidad social de las empresas en un mundo global. Barcelona, Anagrama, 181.
} 
La RSE es pues la aportación empresarial al desarrollo sostenible en sus tres ámbitos, el económico, el social y el medioambiental. Como generadoras de empleos y riqueza, las empresas contribuyen a satisfacer las necesidades de las personas de manera durable (ámbito económico); respetan los derechos humanos en toda la cadena de suministro y favorecen de este modo el bienestar social (ámbito social); por último, contribuyen a la sostenibilidad del planeta a través, entre otras cosas, de la reducción de dióxido de carbono en todas sus actividades (ámbito medioambiental).

Dada la importancia que las empresas otorgan a sus grupos de interés, las corporaciones hacen un esfuerzo particular por tenerlos al día y hacerles partícipes de sus logros en materia de RSE a través de la publicación de informes anuales.

$$
* * *
$$

En el presente artículo analizamos y comparamos los informes anuales de responsabilidad social corporativa de dos empresas. Después de situar la RSE en el contexto más amplio del desarrollo sostenible y de explicar nuestro plan de trabajo y objeto de estudio, pasamos a analizar, por este orden, la memoria de la Corporación RTVE y la de Atresmedia; en el último apartado presentamos nuestras conclusiones.

\section{La RSE y el desarrollo sostenible}

La responsabilidad social de una empresa o corporación consiste en contribuir al desarrollo sostenible para hacer viable el crecimiento económico con el uso de los recursos naturales limitados del planeta y el bienestar y progreso sociales de las generaciones futuras. Como hemos dicho, los organismos internacionales han sido los principales actores del avance y el progreso globales en materia de desarrollo sostenible a finales del siglo XX. La Organización de las Naciones Unidas, especialmente la Organización Internacional del Trabajo (OIT), la Organización para la Cooperación y el Desarrollo Económicos (OCDE) y la Unión Europea (UE), entre otros, han promovido en el ámbito empresarial un entramado de colaboración entre la sociedad civil empresas y ciudadanos - y los poderes públicos para inducir un desarrollo sostenible.

El Pacto Mundial de las Naciones Unidas, adoptado en 2000, es una iniciativa voluntaria basada en el diálogo social para armonizar los intereses de las empresas con las demandas de la ciudadanía en el marco de la economía global. Siendo un instrumento de adhesión voluntaria entre entidades empresariales, sindicatos y organizaciones no gubernamentales $(\mathrm{ONG})$, cuenta con diez principios a implantar en las actividades cotidianas de toda corporación. Además, promueve el compromiso de rendir cuentas a la sociedad mediante una publicidad transparente de los progresos que realiza en consonancia con el citado decálogo.

Los diez principios se basan en declaraciones y convenciones universales y abarcan cuatro áreas: derechos humanos, medio ambiente, normas laborales y anticorrupción. En resumen, las empresas que apoyen la iniciativa deben respetar los derechos humanos fundamentales reconocidos universalmente (área de derechos humanos); deben apoyar la libertad de asociación y el reconocimiento efectivo del derecho a la negociación colectiva; apoyar la erradicación del trabajo infantil; apoyar la abolición 
de las prácticas de discriminación en el empleo y la ocupación (área de normas laborales); deben mantener un enfoque preventivo que favorezca el medio ambiente; fomentar iniciativas que promuevan una mayor responsabilidad ambiental; favorecer el desarrollo y la difusión de las tecnologías respetuosas con el medio ambiente (área de medio ambiente); y deben trabajar en contra de la corrupción en todas sus formas, incluidas la extorsión y el soborno (área anticorrupción).

La RSE se regula mediante otras iniciativas, marcos y directrices nacionales e internacionales - en muchas ocasiones, compartiendo alianzas estratégicasentre las que destacamos las siguientes: SA 8000, certificación voluntaria creada por Social Accountability International (SAI), para garantizar mejores condiciones laborales ${ }^{6}$; Global Reporting Initiative (GRI), que promueve el uso de las memorias de sostenibilidad mediante estándares de información y métodos para medir los impactos y resultados relacionados con la sostenibilidad ${ }^{7}$, y finalmente ISO 26000, que proporciona orientación a las empresas y organizaciones para convertir los principios de responsabilidad social en acciones concretas. Si bien ISO 26000 no certifica como otras normas ISO, incluye el principio de transparencia que define como «la apertura respecto a las decisiones y actividades que afectan a la sociedad, la economía y el medio ambiente y la voluntad de comunicarlas de manera clara, exacta, oportuna, honesta y completa ${ }^{8}$. En esta misma dirección se pronuncia la Norma SGE 21 señalando la importancia de «gestionar para comunicar», pues en último término «no hay buena comunicación de RSE si no se sostiene en un sistema de gestión riguroso y bien integrado en la estrategia de la organización, que se traslada de manera transparente» ${ }^{9}$.

\subsection{La comunicación de la RSE}

Ciertamente, la responsabilidad social forma parte del discurso público de las empresas; de hecho, muchas entidades gestionan la RSE desde sus departamentos de comunicación o de marketing. La comunicación es una herramienta clave en el diálogo con los stakeholders cuando permite establecer canales permanentes de interlocución, a través de iniciativas como la creación de sitios web, asociaciones, proyectos y otro tipo de actividades. Al mismo tiempo, es una forma de consolidar los valores de identidad de la empresa como marca cuando la comunicación (corporativa y comercial) está

${ }^{5}$ El número de empresas de la Unión Europea que han suscrito el Pacto Mundial de las Naciones Unidas ha aumentado de 600, en 2006, a más de 1900, en 2011, según cifras de la Comisión Europea disponibles en: http://eur-lex.europa.eu/LexUriServ/LexUriServ.do?uri=CO M:2011:0681:FIN:es:PDF.

${ }^{6}$ Cuenta con un glosario para interpretar los términos normativos al que se accede en: http:// www.sa-intl.org/index.cfm?fuseaction=Page. ViewPage\&PageID=937.

${ }^{7}$ Asesora a las empresas para elaborar memorias de sostenibilidad de forma transparente. Se puede ver en: https://www.globalreporting.org/languages/spanish/Pages/default.aspx.

${ }^{8}$ El contenido de estas acciones se puede revisar en: https:/www.iso.org/obp/ui/\#iso:std: iso:26000:ed-1:v1:es.

${ }^{9}$ Promueve la inclusión de puntos de contacto para solventar las dudas que puedan surgir sobre el contenido de la memoria. Véase en http://foretica.org/gestionar_para_comunicar_ sge21_g4.pdf 
integrada en el conjunto de estrategias de la empresa, como señala Benavides ${ }^{10}$. Dado que el departamento de comunicación no es el único responsable de la gestión de la imagen corporativa de la organización, la tarea corresponde también a la alta dirección, y exige tanto el compromiso de las distintas áreas funcionales como de cada uno de los empleados, añade Mínguez ${ }^{11}$.

El informe corporativo o memoria es uno de los recursos de comunicación más extendidos para transmitir a la opinión pública los logros de las empresas como entidades responsables con la sociedad. Generalmente este informe muestra el resultado e impacto económico, social y medioambiental de la actividad de la organización que lo realiza en un período de tiempo determinado a través de indicadores cualitativos y cuantitativos. Las recomendaciones sobre el contenido y forma de las memorias tienen como fin último propiciar una opinión fundamentada, ya que deben «contener datos cuantificables, contrastables y verificables, y se deben expresar de forma objetiva e imparcial» ${ }^{12}$. Sin embargo, en la práctica, los informes tienden a comunicar las principales iniciativas de la entidad de cara a resaltar sus actuaciones sin que haya lugar a críticas ni a análisis comparados ${ }^{13}$. Tanto el exceso de elogio como el sesgo en el juicio desfavorable de hechos y conductas hacen visible la cultura corporativa de la organización, que, como ha estudiado Villafañe, conforma la manera de entender el negocio o la actividad básica de la organización y también sus relaciones con el entorno; en este sentido, «es una suerte de atmósfera que envuelve y condiciona la vida corporativa $\gg^{14}$.

La percepción de las prácticas que una organización realiza en materia de responsabilidad social puede producir efectos en su reputación y promover una mayor confianza pública. El Monitor Español de Reputación Corporativa (MERCO), publicado desde 2001, evalúa la reputación de las empresas, la de sus líderes, los entornos laborales y las compañías más responsables y con mejor gobierno corporativo. Difunde diferentes clasificaciones obtenidas mediante la elaboración de una encuesta de opinión entre directivos de empresas, de acuerdo con una visión que tiene en cuenta seis variables: los resultados económicos financieros, la calidad del producto o servicio, la reputación interna, la ética y responsabilidad social corporativa, la dimensión internacional de la empresa y la innovación.

${ }^{10}$ Benavides, J. (2008): «Los grupos de interés y la gestión de valores de responsabilidad social en las empresas y organizaciones», en BAJO, A. y VILLAGRA, N. (ed.), Reflexiones en torno a la responsabilidad social en el ámbito de la globalización. Memoria académica curso 2007-2008. Madrid, Universidad Pontificia de Comillas, 114.

${ }^{11}$ Mínguez, N. (2000): «Un marco conceptual para la imagen corporativa», ZER Revista de Estudios de Comunicación, 8, Bilbao, UPV/EHU, 321. Disponible en: http://www.ehu.es/zer/ es/hemeroteca/articulo/un-marco-conceptual-para-la-imagen-corporativa/115.

${ }^{12}$ VV.AA. (2007): «Informe del Foro de Expertos en Responsabilidad Social de las empresas», Ministerio de Empleo y Seguridad Social, 17, en: http://www.empleo.gob.es/es/sec_trabajo/ autonomos/economia-soc/resposocempresas/foro_expertos/index.htm.

${ }^{13}$ Villagra, N. (2008): «La RSC, su comunicación y la necesidad de una nueva conciencia», en Bajo, A. y Villagra, N. (ed.), op.cit., 133.

${ }^{14}$ Villafañe, J. (2004): La buena reputación. Claves del valor intangible de las empresas. Madrid, Pirámide, 58. 
Los resultados del MERCO 2013 sitúan a Inditex, Mercadona y Santander como las tres empresas españolas con mejor reputación corporativa. El sistema de comunicación utilizado prioritariamente por las dos primeras para transmitir sus mensajes es «el boca a oreja, lo que pone de relieve la importancia que ha alcanzado el ciudadano corriente como predictor» ${ }^{15}$. Además, en los resultados del MERCO 2013 se destaca la dimensión que están adquiriendo las tendencias de reputación online, puesto que están trasladando al espacio de la red lo que anteriormente se situaba en el ámbito de los líderes y de los medios. MERCO 2013 resalta que los usuarios de internet dan más credibilidad a los comentarios que publican los consumidores que a las propias informaciones que transmiten las compañías.

Si consideramos los resultados MERCO durante el período 2001-20013, se observa el crecimiento de la mención de los resultados económicos para considerar una empresa reputada. A partir de 2011, la variable Ética y Responsabilidad Corporativa es menos mencionada a la hora de definir los aspectos clave de la reputación de las compañías. Se identifica entonces la necesidad de contar con una herramienta que evalúe la RSC per se, además del gobierno corporativo, y se crea Merco Responsabilidad y Gobierno.

\subsection{La RSE en las empresas de comunicación}

Las empresas de comunicación están implantando procedimientos de autorregulación de la responsabilidad social como los códigos éticos, los comités de ética o las memorias de sostenibilidad. Sin embargo, en muchas ocasiones, las medidas destinadas a fomentar las actitudes éticas en las corporaciones de comunicación se ponen en práctica en un ámbito de confusión, es decir, su grado de legitimidad es ciertamente una pretensión idílica, si tenemos en cuenta los cuatro criterios de evaluación enunciados por Pava y Krausz: «el grado de conocimiento del problema, el nivel de responsabilidad directa de la empresa, el grado de consenso de todos los grupos sociales y la relación con el resultado económico» ${ }^{16}$.

La empresa de comunicación tiene la misión de conseguir beneficios, ya sean de carácter social o económico. La rentabilidad social atañe, esencialmente, a las empresas de titularidad pública, mientras que la rentabilidad económica acostumbra a asociarse al objetivo de las empresas de comunicación surgidas por iniciativa privada. Sin embargo, en ambos casos los beneficios económicos no deben pasar a un segundo plano, ya que, como indica Mateos-Aparicio, «una pérdida continuada de ganancias es una demostración de una actuación empresarial irresponsable» ${ }^{17}$.

De otro lado y teniendo en consideración estudios sindicales originados en el proyecto de investigación internacional The combating inequality project, promovido

${ }^{15}$ LÓPez Triana, I. (2013): «Observatorio Global de Intangibles», en Villafañe, J. (dir.), La comunicación empresarial y la gestión de los intangibles en España y Latinoamérica. Informe anual 2013. Madrid, Pearson Educación, 7.

${ }^{16}$ Mateos-Aparicio, P. (2007): Dirección y objetivos de la empresa actual. Madrid, Centro de Estudios Ramón Areces, 165.

${ }^{17}$ Ibídem, 167. 
por Global Labour University (GLU) ${ }^{18}$ para impulsar la igualdad social en el marco de la globalidad, la visión sobre los beneficios es bien diferente. Así estos estudios defienden que el crecimiento ya no solo es necesario, sino que tiene que ser un crecimiento integrador, que debe ser respaldado tanto por el sector público como por las empresas privadas. Si los beneficios pueden generarse mediante una gestión que aporte valor a la sociedad, los servicios públicos procuran un efecto positivo en el logro de la igualdad social, defienden.

Con todo, el debate sobre las reformas del sector público se ha centrado en cuestiones de eficacia. «El principio según el cual el sector privado puede proveer servicios de manera más eficiente ha inspirado programas extensos de privatización y mercantilización en los gobiernos de muchos países, impuestos, en parte, por el Banco Mundial y la Unión Europea», observa Hermann ${ }^{19}$. El sector público debe percibirse como un activo económico, como un promotor de justicia social y «no como un lastre sobre la economía privada», por cuanto deben considerarse tanto sus beneficios cuantitativos como cualitativos, añade.

Por su parte, los enfoques deontológicos defienden que el beneficio debe tener como fin el bien común, es decir, aquello que afecta al conjunto de las condiciones de la vida social que «permite a los grupos, y a cada uno de sus miembros, conseguir más plena y fácilmente su propia perfección $»^{20}$. Asimismo, afirman que las empresas de comunicación deben ir más allá de los deberes económicos y de servicio público que les exigen trabajar para satisfacer los intereses de los usuarios destinatarios de la información, y les piden que reflejen una actuación socialmente sensible, acorde con la misión de la empresa y los procesos productivos

En este contexto, nos preguntamos si las empresas del sector de la comunicación comunican mejor sus logros en materia de responsabilidad social que las que se dedican a otras actividades; si su especialización es un valor añadido a la hora de contactar con los grupos de interés; también si es distinto informar, de manera transparente, sobre una empresa pública que hacerlo sobre una empresa privada.

\section{Plan de trabajo y objeto de estudio}

A priori, sabemos que los medios de comunicación están especializados en informar y que participan de manera crucial en la formación de la opinión pública, pero desconocemos si son capaces de aplicarse lo que predican. Como empresas que son, las compañías de comunicación emiten informes corporativos, rinden cuentas ante sus respectivos consejos de administración, quieren estar en contacto con sus clientes,

\footnotetext{
${ }^{18}$ Red de instituciones de investigación y de centros sindicales nacionales e internacionales con sede en Alemania, Brasil, India y Sudáfrica, apoyada por la Oficina Internacional del Trabajo (OIT).

${ }^{19}$ Hermann, C. (2014): «El papel del sector público en el combate de la desigualdad», Boletín Internacional de Investigación Sindical, vol. 6, núm. 1. Ginebra, Oficina Internacional del Trabajo, 142. Disponible en: http://www.ilo.org/wcmsp5/groups/public/---ed_dialogue/--actrav/documents/publication/wcms_247984.pdf.

${ }^{20}$ Polo, A. (2008): «El empresario ante la Ley moral», en Bajo, A. y Villagra, N. (ed.), op.cit., 67.
} 
inversores, empleados y, en definitiva, con sus grupos de interés ¿Son transparentes las memorias de responsabilidad corporativa de Atresmedia y de la Corporación RTVE? ¿La titularidad privada o pública de una empresa afecta a la forma de comunicar con sus grupos de interés?

Como decimos, el informe corporativo tiene por objeto transmitir el valor creado por la empresa. En muchas ocasiones, la información facilitada en las memorias anuales se refiere a indicadores generales y está excesivamente fragmentada, aspecto que explica la escasa conectividad entre los datos, tal como ha puesto de manifiesto un estudio de la consultora Price Waterhouse Coopers $(\mathrm{PwC})$ sobre empresas españolas ${ }^{21}$. Frente a la dispersión de datos, $\mathrm{PwC}$ promueve el modelo de información integrado (integrated reporting, IR), fórmula que reúne información relevante sobre la estrategia de la organización, su sistema de gobierno, el desempeño y las perspectivas de futuro, de manera que refleje los impactos económicos, ambientales y sociales de la compañía sobre los entornos en los que opera.

Tomando como referencia las conclusiones del estudio canónico de $\mathrm{PwC}$, nuestro objeto de estudio son las memorias de responsabilidad de la Corporación Radiotelevisión Española (RTVE) y de Atresmedia Corporación de Medios de Comunicación, S.A., correspondientes al ejercicio 2012, para establecer un marco comparativo que posibilite resolver si los informes corporativos de los dos grupos presentan carencias similares en la comunicación de sus logros, o si, por el contrario, ofrecen un grado de transparencia informativa superior al del resto de las empresas, dada su especialización y trayectoria en el ámbito de la comunicación pública.

Para la delimitación del objeto de estudio hemos creído de interés comparar la memoria de una empresa de comunicación de titularidad pública (RTVE) con otra de titularidad privada (Atresmedia). Las tendencias de ambos informes pueden ser reflejo de la manera de entender la comunicación que tienen ambas organizaciones y de las relaciones que establecen con el entorno, a través de sus webs corporativas. Aclaramos que se toma como referencia el periodo $2012^{22}$ porque los informes disponibles en la web, en el momento de realizar nuestro estudio, se refieren a ese año. Se ha descartado la posibilidad de comparar el informe de 2012 de RTVE con el de Atresmedia, relativo a 2013.

La primera conclusión del informe de $\mathrm{PwC}$, que indica que muchas entidades se limitan a publicar información acotada al mero cumplimiento de los requerimientos legales $-\mathrm{y}$, con ello, pierden la oportunidad de comunicar el valor creado-, y a continuación tenemos en cuenta las recomendaciones de mejora que propone el estudio de PwC. En síntesis, las conclusiones de PwC son las siguientes:

a) La información sobre riesgos y oportunidades es insuficiente. La comunicación de las empresas sigue basándose en el marco del cumplimiento para España,

${ }^{21}$ El estudio analiza los informes del ejercicio 2012 de todas las empresas del IBEX 35 y establece una comparativa con los de compañías incluidas en otros índices bursátiles europeos e internacionales. Se puede consultar en: http://www.pwc.es/es_ES/es/publicaciones/auditoria/ assets/pwc-reporting-integrado-2013.pdf.

${ }^{22}$ RTVE no había publicado en su web el informe relativo al ejercicio 2013. El de Atresmedia está en su web corporativa desde junio de 2013. 
pero no aborda temas relevantes como, por ejemplo, cómo puede variar el perfil de riesgos de la empresa en el tiempo y el impacto que pueden tener esos riesgos.

b) La información sobre el desempeño del consejo de administración y sus comisiones durante el ejercicio no permite evidenciar la efectividad de la labor del máximo órgano de gobierno, ni su contribución a la creación de valor.

c) La comunicación de los indicadores de desempeño claves del negocio es prácticamente nula. Se recomienda identificar claramente aquellos indicadores específicos de la empresa, que permitan la medición y seguimiento de los objetivos estratégicos, y vincular las políticas retributivas a indicadores concretos.

d) Los inversores consideran mejorables aspectos como la conectividad y la precisión de la información, la capacidad de mantener la credibilidad y generar una visión de creación de valor en el largo plazo.

Además, en nuestro trabajo planteamos un acercamiento a las nuevas tendencias de comunicación corporativa, definidas por el compromiso y la mayor vinculación (engagement) con los grupos de interés. La confianza y credibilidad de las empresas con sus stakeholders han de considerar los cambios en los comportamientos sociales producidos por las nuevas tecnologías de la información. Por ello, apoyamos nuestra revisión de las dos memorias con los resultados del Barómetro de Confianza de Edelman de $2014^{23}$, que otorga a los medios sociales e híbridos un alto reconocimiento frente a los medios tradicionales y propios. El cotejo de los dos informes corporativos nos revelará si consideran las necesidades y expectativas de los interlocutores que generan más confianza. Esto es, los iguales («alguien como tú y como yo», de acuerdo con la expresión utilizada en el Barómetro) y los empleados, con subidas de 5 y 7 puntos porcentuales respectivamente respecto a 2013, según el Barómetro. Por su parte, las opiniones de los académicos y expertos (internos o externos a la empresa) siguen liderando la confianza, con aumentos del 3 y del 1 por ciento respectivamente en relación al año anterior, frente a aquellos portavoces que ocupan alta posición jerárquica en la empresa (junta directiva), que han registrado un descenso del 6 por ciento en relación a 2013.

Proponemos, entonces, el siguiente modelo de estudio: siguiendo la monografía de PwC, situamos los informes corporativos de RTVE y Atresmedia en el contexto de las empresas españolas que cotizan en el IBEX para establecer la competencia de sus reportes, y utilizamos las recomendaciones del Barómetro de Confianza de Edelman relativas a España para aplicar criterios de reputación que tengan en cuenta

${ }^{23}$ Véase 2014 Edelman Trust Barometer. Resultados para España. La confianza global en los medios de comunicación vuelve a niveles de 2010. Casi el 80 por ciento de los países informan de que confían menos en los medios de comunicación. Accesible en: http://www.edelmanspain. es/. 
la participación, conectividad, movilidad y relación entre las personas que ofrecen los medios sociales.

Centraremos nuestro interés en la accesibilidad y facilidad de navegación por los informes disponibles en las webs corporativas, en su claridad expositiva y en la información facilitada sobre los empleados. Los datos económicos facilitados no son en ningún caso objeto de nuestro análisis, aunque abordaremos si estos indicadores presentan sesgos y ambigüedades que propicien un diálogo incompleto con los grupos de interés.

Las figuras que hemos elaborado se presentan bajo los epígrafes y categorías profesionales utilizadas por los dos grupos de comunicación en sus respectivas memorias. Somos conscientes de que las denominaciones responden a clasificaciones procedentes de su diferente titularidad, como sabemos pública y privada.

Estimamos que la puesta en relación de las memorias de responsabilidad de RTVE y de Atresmedia puede resultar interesante para visibilizar la cultura corporativa de estos dos grupos de comunicación.

\section{La memoria de la Corporación RTVE}

En el caso de la Corporación RTVE, el informe estudiado es «Memoria de Servicio Público y Responsabilidad Social Corporativa», de fecha 26 de mayo de 201424, correspondiente al ejercicio 2012 y disponible en la web corporativa http://www.rtve. es/corporacion/rc/, en la rúbrica «Corporación RTVE». El documento ${ }^{25}$ contiene 367 páginas y está articulado en los cuatro títulos siguientes: «Así somos», «Consecuentes con el presupuesto», «Comprometidos con la sociedad» y «Gracias a vosotros nos reconocen por nuestro trabajo».

La memoria va precedida de una carta del presidente de la corporación ${ }^{26}$, rubricada, pero sin pie de firma, y de un índice, sin paginar, donde se expone la lista ordenada de los asuntos tratados.

Con el título «Así somos», se dedican 18 páginas a informar sobre los principios generales que marcan la actuación de RTVE y los cinco textos legales de donde emanan sus principios básicos en responsabilidad corporativa. Además, se incluyen las áreas de actividad de la corporación y las marcas que la integran para la gestión del servicio público de radio, televisión y web de titularidad del Estado, a través de dos sociedades mercantiles estatales cuyas acciones son propiedad de la corporación: Televisión Española (TVE) y Radio Nacional de España (RNE). TVE, compuesta por La 1, La 2, Teledeporte, Clan, Canal 24 Horas y TVE Internacional; RNE, integrada por RNE (Radio 1), Radio 3, Radio 4, Radio 5, Radio Clásica y Radio Exterior de España; el portal de la corporación RTVE.es, además del Instituto RTVE y la Orquesta y Coro de RTVE.

\footnotetext{
${ }^{24}$ Se trata de la actualización de la página web, si bien el documento se publicó el 9 de febrero de 2014.

${ }^{25}$ En aras de la brevedad, lo denominamos Memoria RTVE.

${ }^{26}$ Leopoldo González-Echenique ha ocupado este cargo desde julio de 2012 hasta septiembre de 2014.
} 
Asimismo, se da cuenta de los informes de las comisiones del consejo de administración, así como de los nombres del nuevo presidente y consejeros. El texto señala que durante el período de referencia «se eliminaron las retribuciones fijas de los consejeros, a excepción del presidente, siendo sustituidas por indemnizaciones por asistencia a las sesiones del Consejo», y que «siguiendo esta misma línea de ahorro, se eliminaron también los puestos de asesores y secretarias de los consejeros» ${ }^{27}$. (No se ofrece información sobre la cuantía de estas indemnizaciones, ni de la cantidad resultante de la eliminación de asesores y secretarias.)

Sobre la actividad de la comisión de servicio público, la memoria refiere dos reuniones mantenidas durante 2012 centradas en la adaptación de la señalización de los contenidos televisivos, según tramos de edad, y en la recomendación de la defensora del telespectador para que los responsables de la programación cuidasen y mantuviesen la «magia de los Reyes Magos $»^{28}$, que había sido puesta en duda en las quejas cursadas por los telespectadores. Igualmente, detalla la modificación de varios artículos del manual de estilo de la Corporación, relativos a errores gramaticales, y otros asuntos de trámite centrados en el plan de auditoría correspondiente a 2012.

\subsection{Financiación sin publicidad}

Bajo el título «Consecuentes con el presupuesto», de otras 18 páginas, se informa de la entrada en vigor de la Ley 8/2009, de 28 de agosto de 2009, y del establecimiento de un nuevo sistema de financiación para la Corporación RTVE y sus sociedades dependientes que ha supuesto la supresión de la publicidad como fuente de financiación. En este apartado, se da cuenta de la ejecución presupuestaria y del balance de cuentas. Los datos de cierre del 2012 se comparan con el presupuesto oficial aprobado por el Parlamento para el ejercicio. El seguimiento se realiza de acuerdo con los conceptos de consumos, gastos de personal, tributos, amortizaciones, dotación por deterioro y otros gastos.

En la sección de inversiones, destaca el proceso permanente de inversión en nuevas tecnologías para aplicar a las áreas de Sistemas, Ingeniería y Distribución de contenidos.

El título «Comprometidos con la sociedad», de 341 páginas, es el de mayor extensión. En la primera parte, hace hincapié en el objetivo de llegar a todos los públicos mediante una programación responsable, tanto en TVE como en RNE, y a través de los medios interactivos. Explica igualmente la obligación contraída con la formación (desde el Instituto RTVE) y la música (con la Orquesta y Coro de RTVE). Asimismo, incluye secciones relativas a fortalecer las relaciones internacionales, con el propósito de lograr ser la primera cadena del mundo en lengua española; la participación del público, por medio de la oficina de la defensora del espectador; la conservación del fondo documental y los archivos históricos, y la innovación tecnológica para asegurar la gestión y supervisión de las emisiones y los equipamientos de producción. Por último, refleja las actuaciones en proyectos de ahorro, accesibilidad y otros vinculados

\footnotetext{
${ }^{27}$ Memoria RTVE, 18.

${ }^{28}$ Ibídem, 20.
} 
bajo el subtítulo «Responsabilidad Social Corporativa».

La serie de indicadores continúa como sigue: las campañas institucionales realizadas; la participación en procesos electorales y campañas sociales; la programación responsable con los derechos laborales; la innovación, la marca España, la cooperación y la solidaridad; integración, pluralidad, educación y formación, igualdad, personas mayores, salud y prevención, medio ambiente, sostenibilidad y transparencia.

La última parte de esta sección está dedicada a los recursos humanos. Los datos aportados en la memoria indican que a finales de 2012 la plantilla ascendía a 6.319 trabajadores - 5.781 empleados fijos, 438 contratados y 99 empleados indefinidos, con una media de edad de 44,5 años y un promedio de antigüedad de 18 años-. (En este desglose, se observa un error en el cálculo: falta un trabajador para llegar a los 6.319.)

La mayoría de la plantilla está adscrita a TVE - con 3.565 efectivos—-, seguida de RTVE, con 1.912, de RNE, con 704, y de RTVE.es, con $138^{29}$.

Entre las mejoras implementadas, la memoria destaca nuevas funcionalidades de «El Portal del Trabajador», de cara a facilitar la comunicación interna, y la ejecución de dos encuestas; una para evaluar la satisfacción de los empleados con el servicio de comedor, y otra para conocer la opinión de los trabajadores sobre la propuesta del director de los Servicios Informativos de TVE para aplicar un método electrónico de votación que garantice la confidencialidad del voto. (No se informa de los resultados de ninguna de estas dos consultas.)

La memoria reseña las elecciones sindicales realizadas el 17 de noviembre de 2011 y de la representación sindical en el comité (Intercentros) de la Corporación RTVE, integrado por cuatro representantes de UGT, tres de CC.OO., tres de Alternativa APLI, uno de Alternativa RTVE y uno de USO.

\section{2 Áreas con desequilibrios de género}

En cuanto al compromiso con la igualdad de género, la memoria informa del Plan de Igualdad de la Corporación ${ }^{30}$, destinado a desarrollar políticas que integren la igualdad de trato y de oportunidades entre hombres y mujeres. Para garantizar la representación equilibrada, se modificó el texto del I Convenio colectivo de la Corporación y, en relación a la política de contratación, se dispuso que «a igualdad de méritos y competencia, acceda al puesto la persona del sexo menos representado» ${ }^{31}$.

En la figura 1 se resumen los porcentajes que revelan «desequilibrios en la proporción de hombres y mujeres», fundamentalmente, en las áreas Técnica y de Emisiones de RNE, Sistemas, Medios TVE y Recursos Humanos y Organización ${ }^{32}$.

En la revisión de datos realizada destacamos la alta proporción de hombres en las áreas técnicas y la de mujeres en los puestos administrativos, estas últimas con un porcentaje del 74 por ciento. En los puestos de dirección, los desequilibrios se evidencian con valores similares: el 73 por ciento son hombres, y en el consejo de administración

\footnotetext{
${ }^{29}$ Memoria RTVE, 310.

${ }^{30}$ Publicado en el BOE el 12 de julio de 2012.

${ }^{31}$ Memoria RTVE, 316.

${ }^{32}$ Ibidem, 317.
} 
la presencia de mujeres es del 33 por ciento; si bien estos últimos porcentajes no se proporcionan en la memoria - se facilitan cantidades globales-, los hemos calculado e incluido en la figura 1 para que todos los indicadores se ajusten a un mismo criterio.

Fig. 1 Plantilla de RTVE 2012

\begin{tabular}{|c|c|c|}
\hline Áreas & Mujeres & Hombres \\
\hline Dirección & $27 \%$ & $73 \%$ \\
\hline & $33 \%$ & $67 \%$ \\
\hline Consejo de Administración & $38 \%$ & $62 \%$ \\
\hline TVE & $13 \%$ & $87 \%$ \\
\hline $\begin{array}{c}\text { Área Técnica } \\
\text { y Emisiones RNE }\end{array}$ & $21 \%$ & $79 \%$ \\
\hline Sistemas & $23 \%$ & $77 \%$ \\
\hline Medios TVE & $74 \%$ & $26 \%$ \\
\hline RRHH y Organización & $40 \%$ & $60 \%$ \\
\hline Total plantilla & & \\
\hline
\end{tabular}

Fuente: Elaboración propia con datos de la Memoria RTVE 2012.

Para finalizar, se relatan las campañas de salud emprendidas entre los trabajadores y la política en prevención de riesgos laborales poniendo de relieve el cumplimiento y el reconocimiento oficial Ohsas $18001^{33}$.

El último título - «Gracias a vosotros nos reconocen por nuestro trabajo»-consta de 22 páginas y da cuenta de los premios concedidos durante 2012 a la Corporación RTVE y a las marcas que la integran. El palmarés de premios y festivales de TVE es el más extenso e incluye todo tipo de galardones, ya sean a título individual, como los Premios Ondas Nacionales a los presentadores Anne Igartiburu (La 1) y Jordi Hurtado (La 2), ya sean series de ficción como «Águila Roja», finalista en los XIV Premios Academia de las Ciencias y las Artes de Televisión (ATV). Otros, como el Premio Comité Paralímpico Español a la cadena, por el apoyo al deporte de discapacitados, o el recibido por el consejo de Informativos de TVE - el VIII Premio José Couso de Libertad de Prensa - valoran al conjunto de equipos profesionales.

Junto a la memoria objeto de estudio, y dentro también de la rúbrica «Responsabilidad Corporativa» ${ }^{34}$, hay otro documento denominado «Informe de Progreso 2012 Pacto Mundial ${ }^{35}$-igualmente fechado el 26 de mayo de 2014- que resume la citada memoria y resalta las campañas realizadas mediante acuerdos con el UNICEF, la FAO y otras organizaciones que trabajan por la defensa de los derechos humanos y en diferentes causas en consonancia con los Objetivos de Desarrollo del Milenio y el Pacto

${ }^{33}$ Certificación de AENOR que facilita la integración de sistemas de gestión de calidad, ambiental y de seguridad y de salud en el trabajo, entre otros.

${ }^{34}$ También figuran las memorias correspondientes a los años 2008, 2009, 2010 y 2011.

${ }^{35}$ RTVE se incorporó a la Red Española del Pacto Mundial en julio de 2011. 
Mundial. En aras de la transparencia, el informe se publica en la intranet de RTVE, se presenta en el Parlamento y, posteriormente, se entrega a los medios de comunicación.

Además, RTVE anuncia una convocatoria de «Cesión de bienes equipos», para ampliar la vida útil de equipos informáticos y técnicos - en perfecto estado de funcionamiento- cediéndolos a entidades sin ánimo de lucro que apoyen preferentemente proyectos de cooperación, desarrollo e interés social ${ }^{36}$.

\section{El informe de Atresmedia}

Atresmedia titula su memoria «Informe Anual y de Responsabilidad Corporativa 2012». Se trata de un documento ${ }^{37}$ de 177 páginas referido al período comprendido entre el 1 de enero y el 31 de diciembre de 2012, disponible en la web corporativa ${ }^{38}$, accesible a través de la rúbrica «Informes $\mathrm{RC}$ » y con entrada también desde un menú lateral $^{39}$.

En el sitio web figuran otros accesos con ingreso a «Políticas corporativas», «Códigos de conducta» — se incluye el código de conducta de Atresmedia, el código deontológico de los Servicios Informativos de Antena 3 y el código deontológico de La Sexta Noticias - y a la denominada «Zona de descargas», con treinta documentos parciales del informe anual y otros tres referidos a certificaciones.

El informe recoge de manera unificada los aspectos financieros y no financieros (sociales, ambientales y de buen gobierno) de la sociedad resaltando en color verde toda la información relativa a responsabilidad corporativa.

El índice, paginado, da cuenta de los asuntos a tratar incluyendo en las primeras páginas una carta firmada por el presidente de Atresmedia — José Manuel Lara - y un mensaje rubricado por el consejero delegado - Silvio González-. A continuación, se articulan los doce títulos siguientes: «Principales magnitudes de Atresmedia», «Lo más destacado del año», «Atresmedia», «Atresmedia Televisión», «Atresmedia Radio», «Atresmedia Cine», «Atresmedia Digital», «Atresmedia Publicidad», «Recursos Humanos», «Sociedad», «Medio Ambiente» y «Anexos».

La información objeto de estudio cubre todas las actividades de Atresmedia: televisión (integrada por Antena 3, laSexta, Neox, Nova, Nitro, xplora y laSexta3 (Gol TV en régimen de alquiler)), radio (Onda Cero, Europa FM y Melodía FM), Atresmedia Cine, Atresmedia Publicidad y la Fundación Antena 3.

Las principales magnitudes de Atresmedia se agrupan en función de tres enfoques: económico, social y ambiental, y se presentan en un cuadro que ocupa una sola página y que recopila los resultados de la corporación comparándolos con los ejercicios 2010 y $2011^{40}$.

\footnotetext{
${ }^{36}$ A entidades cuyos objetivos sean acordes a la ley 17/2006, de 5 de junio, de la radio y la televisión de titularidad estatal.

${ }^{37}$ En aras de la brevedad, lo denominamos Informe Atresmedia.

${ }^{38} \mathrm{http}$ ://www.atresmediacorporacion.com/responsabilidad-corporativa/informes/.

${ }^{39}$ Junto a los informes de los ejercicios 2013, 2011, 2010, 2009, 2008 y 2007.

${ }^{40}$ Informe Atresmedia, 13.
} 
En la dimensión económica se incluyen indicadores sobre ingresos netos totales, resultado bruto de explotación, beneficio neto, valor económico distribuido, ayudas económicas recibidas de la administración pública, ratio de eficiencia publicitaria (diferenciada en adultos e individuos) y número de anunciantes, cuota media de pantalla de Antena 3 Televisión, Neox, Nova, Nitro, laSexta, xplora y laSexta3, share y número de oyentes de Onda Cero y Europa FM, y datos sobre el número de vídeos servidos a través de antena3.com, lasexta.com y xplora.com.

La dimensión social está integrada por las cifras relativas a horas de programación infantil emitidas (crecientes) y horas acumuladas con subtítulos (crecientes); número de empleados (decreciente); inversión en formación (decreciente) y ratio de horas de formación por empleado (decrecientes); los ingresos de la Fundación aportados por Atresmedia (crecientes); incumplimientos del código de autorregulación (decrecientes), e indicadores sobre costes de oportunidad por la emisión de espacios publicitarios sociales sin coste en radio y televisión (sin información relativa a 2010).

En la dimensión medioambiental, el cuadro presenta el consumo de papel, tóner y cartuchos de impresión (con resultados decrecientes); de luces fluorescentes, residuos sólidos urbanos, gas, gasóleo (calefacción) (en descenso); de electricidad y agua (crecientes en 2012); de emisiones de gases de efecto invernadero (GEI) (decrecientes), así como la inversión ambiental, con un fuerte descenso.

\subsection{La integración de laSexta}

El título «Lo más destacado del año», de 10 páginas, vuelve a incidir en las magnitudes principales de Atresmedia señalando la bonanza de los resultados obtenidos «en un entorno económico de caída del mercado publicitario y de debilidad a nivel macroeconómico» ${ }^{41}$. El informe subraya el éxito en la integración de laSexta, a propósito de la fusión con Antena 3, acaecida el 1 de octubre de 2012, hecho fundamental durante el período objeto de estudio y que confirma a Atresmedia como propuesta líder en el sector de la televisión, con ocho canales, y la consolida como grupo de comunicación líder contabilizando su presencia en los mercados publicitarios de radio y de cine.

El título «Atresmedia», de 28 páginas, es el de mayor extensión. Primeramente, describe la misión, visión y valores del grupo; la estructura accionarial y la composición del consejo de administración (incluye fotografía de los integrantes), la de sus comisiones y la del equipo directivo. Seguidamente, pasa a exponer datos sobre el entorno económico durante el ejercicio: la evolución del IBEX - la acción de Antena 3 Televisión cerró en 3,90 euros, cayendo un 16,13 por ciento en el año-, la del resto de plazas europeas y los índices registrados en el mercado estadounidense, la recesión de la economía española, el aumento del desempleo, la caída del consumo en los hogares españoles y las previsiones a la baja para el ejercicio 2013. «A pesar de los débiles resultados del mercado, Antena 3 Televisión mantiene su compromiso con los accionistas, y pagará cerca de un 70 por ciento del Beneficio Neto en concepto de dividendo $\rangle^{42}$.

${ }^{41}$ Informe Atresmedia, 15.

${ }^{42}$ Ibídem, 32. 
A continuación, el informe detalla la atención de la compañía a accionistas e inversores, mediante el departamento de Relación con Inversores (RI) y la Oficina del Accionista, que ha establecido más de 500 contactos.

Después se reseñan las acciones del Plan Director de Responsabilidad Corporativa 2010-2012 para mejorar las relaciones de confianza entre la compañía y sus grupos de interés prioritarios, esto es, anunciantes, audiencias, sociedad, empleados, competidores, organismos reguladores, proveedores y accionistas. Entre las mejoras implementadas se da cuenta de los siguientes canales de interlocución: la página web corporativa, el canal telefónico — que ha atendido 29.000 llamadas con dudas, consultas, solicitud de información u opiniones-, un focus group, destinado fundamentalmente a conocer la opinión de los empleados, y un reporte en sostenibilidad dirigido a analistas e inversores, con indicadores de la valoración de prácticas y desempeño en materia social, medioambiental y ética realizados por diferentes entidades de evaluación. Se detalla, además, la participación de Atresmedia en distintos foros de responsabilidad social y su colaboración con universidades para impulsar el conocimiento en materia de RSE.

En el apartado dedicado a la «Prevención y control de riesgos corporativos», se informa de que la compañía ha establecido un completo sistema para la gestión y prevención de riesgos corporativos, basado en la identificación de los mismos y el establecimiento de medidas de control y protocolos de actuación $\rangle^{43}$. (No se expone el perfil de estos riesgos, ni el impacto que pueden tener.)

Los títulos siguientes — «Atresmedia Televisión», «Atresmedia Radio», «Atresmedia Cine», «Atresmedia Digital» y «Atresmedia Publicidad»- ocupan 23 páginas, informan del crecimiento de la audiencia y del acierto en los estrenos en todas las parrillas de programación. Resumimos aquí algunos de los titulares: «Las cadenas de Atresmedia Televisión se imponen a sus directas competidoras»; «Onda Cero, el mejor resultado de los últimos once años»; «Europa FM, de nuevo, la cadena musical que más crece»; «Herrera en la Onda, el gran referente de las mañanas»; «Julia en la Onda amplía su ventaja»; «La Brújula, el segundo informativo de noche más escuchado»; «Te doy mi palabra, la oferta más atractiva del fin de semana»; «Éxito de taquilla y crítica»; «A la vanguardia de la interactividad»; «Liderazgo en las redes sociales»; «La integración con laSexta: el nacimiento de una nueva oferta comercial» $\mathrm{y}$ «Publicidad comprometida y de calidad», entre otros.

\subsection{Recursos humanos}

El informe dedica 15 páginas a los recursos humanos. Se indica que a finales de 2012 la plantilla de Atresmedia ascendía a 1.784 personas. Sin embargo, en el gráfico que abre y complementa esta sección se cuantifican 1.773 empleados $^{44}$. (Entre las dos cifras observamos una discrepancia de 11 personas.)

«La disminución de la plantilla durante el año 2012 se ha debido, fundamentalmente, a los cambios organizativos en respuesta a las nuevas necesidades del sector audiovisual

${ }^{43}$ Informe Atresmedia, 46.

${ }^{44}$ Ibidem, 115. 
y que han requerido la externalización de ciertos servicios» ${ }^{45}$. (Para esclarecer el alcance de la disminución de plantilla tenemos dos opciones: bien recurrir al gráfico citado - con la discrepancia detectada — , bien retroceder a la página 13 del documento y recuperar las cifras relativas al número de empleados mostradas en los tres ejercicios; esto es: 1.942 (2010), 1.849 (2011) y 1.784 (2012). Dado que no se ofrece la cuantía, ni el porcentaje del descenso en ningún lugar, procedemos a calcularlo siguiendo las referencias de la página 13. Por tanto, en 2011 hay 93 empleados menos que en 2010, lo que representa un descenso del 4,78 por ciento. Por su parte, en 2012, hay 65 empleados menos que en 2011, lo que representa un descenso del 3,64 por ciento.)

Conociendo que los datos pueden ofrecerse al público desde distintas perspectivas, entendemos que la información facilitada debe ajustarse a criterios de propiedad y precisión. Si en el enunciado se alude una «disminución de la plantilla», es pertinente facilitar la información completa y no dar por hecho que el grupo de interés que lee el informe va haciendo mentalmente todos los cálculos con las informaciones parciales facilitadas, parte en los gráficos, parte en el texto.

El informe continúa relatando que la media de edad de la plantilla es de 40,6 años y que los empleados se localizan fundamentalmente en Madrid (1.475), Andalucía (110), Cataluña (75) y Galicia (69). (La suma de estas localizaciones proporciona un resultado de 1.729 empleados. Detectamos que en el informe hay 55 efectivos sin ubicar. Es posible que se encuentren adscritos a otras comunidades y agrupados en otras localizaciones, pues se añade a continuación que «se dispone de empleados por el resto del país y se facilita la posibilidad de rotación entre ciudades por voluntad propia de los trabajadores $»^{46}$.)

Sobre los contratos, se informa de que el porcentaje de plantilla con contrato indefinido aumentó en 2012 hasta el 84 por ciento (1.490), y que su antigüedad media en la empresa se estableció en 11,5 años. Para consolidar los valores de igualdad y diversidad, durante 2012 se incrementaron los esfuerzos en la elaboración de un plan de igualdad de la compañía, con medidas para garantizar la igualdad y favorecer la conciliación laboral y familiar. (No se concretan las medidas. Sólo se añade que el plan «está pendiente de la aprobación del Comité de Empresa» ${ }^{47}$.)

Desde la perspectiva de género, los principales porcentajes de la plantilla de Atresmedia revelan que el 84,35 por ciento de las mujeres trabajan en puestos administrativos y que los puestos directivos están ocupados principalmente por hombres, estos últimos con una proporción del 70,8 por ciento. Igualmente, los desequilibrios se hacen patentes en el consejo de administración, con un 20 por ciento de participación femenina, si bien el informe no refiere estas cifras en términos de «desequilibrio», sino que destaca la distribución igualitaria por sexos que existe en la compañía, con un 48,6 por ciento de mujeres, y detalla las nuevas incorporaciones producidas durante 2012, con más del 55 por ciento de mujeres ${ }^{48}$.

\footnotetext{
${ }^{45}$ Ibidem, 115.

${ }^{46}$ Informe Atresmedia, 115.

${ }^{47}$ Ibidem, 115.

${ }^{48}$ Ibidem, 115.
} 
En la figura 2 se resumen los porcentajes indicativos de la plantilla de Atresmedia, donde apreciamos cierto equilibrio en la presencia de mujeres en las categorías profesionales técnicas.

(Como en el caso de RTVE, hemos calculado los porcentajes no facilitados para que todos los indicadores se ajusten a un mismo criterio.)

Fig. 2 Plantilla de Atresmedia 2012

\begin{tabular}{c|c|c|}
\hline & & \\
\hline Categoría profesional & Mujeres & Hombres \\
\hline Directivos & $29,1 \%$ & $70,8 \%$ \\
\hline Conseio de Administración & $20 \%$ & $80 \%$ \\
\hline Técnicos & $46 \%$ & $54 \%$ \\
\hline Administrativos & $13 \%$ & $87 \%$ \\
\hline Otros & $39,4 \%$ & $61 \%$ \\
\hline Total plantilla & $48,6 \%$ & $51,4 \%$ \\
\hline
\end{tabular}

Fuente: Elaboración propia con datos del Informe Atresmedia 2012.

En lo relativo a la política salarial, el informe indica que Atresmedia se caracteriza por situar los salarios de los empleados «por encima de lo exigido por convenio y muy por encima de la media del sector». En el caso de los directivos (directores, subdirectores y gerentes), explica que el salario se compone de una parte fija (salario base) y de otra variable ligada a los resultados individuales, a los resultados globales de la compañía y al resultante de la «Evaluación de Competencias $\left.180^{\circ}\right\rangle^{49}$. (Una vez más, no se ofrece ningún indicador salarial de la compañía ni del convenio colectivo de aplicación.)

El título «Sociedad», de 18 páginas, da cuenta de las campañas asumidas en consonancia con la mejora de la seguridad vial, a través de «Ponle Freno»; de la lucha contra la obesidad infantil, mediante la iniciativa «El Estirón», y de la sensibilización, en el respeto al medio ambiente, en especial de los más jóvenes, a través de «Hazte Eco».

La acción social de Atresmedia se realizó por vía de la Fundación Antena 3 impulsando un programa de asistencia hospitalaria desde el canal de televisión Fan3. Para facilitar la incorporación laboral de las personas con discapacidad en el ámbito del sector audiovisual, destaca la continuidad del proyecto PRO, con la creación de un posgrado en comunicación multimedia y la impartición de un nuevo curso del Programa de Técnico en Accesibilidad a la Televisión, con formación en subtitulado para sordos, interpretación de la lengua de signos y audio descripción.

El informe reseña el apoyo a otras campañas sociales como los bancos de alimentos, proyectos del UNICEF, «Crea Cultura» (para concienciar a los espectadores contra la piratería informática); la celebración del «Día SOMOS», en laSexta, con la reunión

${ }^{49}$ Ibidem, 120-121. 
de 25 ONG españolas para dar gracias a los 3 millones de españoles que las apoyan regularmente con sus donativos, y la colaboración de Onda Cero con la Campaña del Milenio de Naciones Unidas, «Y tú sin enterarte», para comunicar los logros alcanzados tras una década de lucha contra la pobreza extrema.

\section{Conclusión}

La revisión de las memorias de responsabilidad corporativa de RTVE y de Atresmedia confirma niveles de transparencia informativa similares a los de las empresas del IBEX 35. Por lo tanto, se confirman las prácticas de comunicación empresarial reveladas por el estudio de PwC. En este sentido, nuestra revisión constata, además, que la especialización en el sector de la comunicación pública de estos dos grupos multimedia no constituye un valor añadido en el diálogo con sus grupos de interés.

Igualmente, las dos compañías proceden por saturación: proporcionando muchos datos, informaciones repetidas y desorganizadas, que inducen a confundir más que a orientar. Dado que los informes de responsabilidad corporativa estudiados omiten indicadores referidos en sus propios enunciados y que se detectan giros repentinos en el punto de vista argumental, es muy difícil que puedan conseguir la credibilidad de sus grupos de interés. Es más, las memorias corporativas de Atresmedia y de RTVE pierden fiabilidad en la medida en que se van detectando los juegos malabares que han ido superando sus redactores para conformar su relato.

Atresmedia y RTVE comparten las siguientes tendencias:

a) Pasan por alto informar sobre riesgos

b) Suprimen la evaluación del desempeño del consejo de administración

c) Omiten indicadores sobre políticas retributivas

d) Facilitan información inconexa que desalienta los análisis comparados

e) Propician la ambigüedad y un diálogo incompleto con los stakeholders

f) Tienen interés en destacar la implantación de políticas de igualdad de género

g) Muestran errores de cálculo en los indicadores relativos a la plantilla

Paralelamente, el marco comparativo nos ha permitido observar que los informes o memorias alojados en la red no consideran las necesidades y expectativas de los interlocutores que generan más confianza, es decir, los iguales y los empleados, de acuerdo con la evaluación del Barómetro de Confianza de Edelman seguido en nuestro plan de trabajo.

El cotejo de los dos informes corporativos nos hace concluir que la memoria de Atresmedia ofrece:

- Mayor accesibilidad, mediante la creación de un microsite específico en la web corporativa que facilita búsquedas aleatorias y descargas. Además, el índice paginado de los asuntos a tratar proporciona una navegación orientada

- Información sobre la identidad de los líderes, esto es, del equipo de dirección y de los miembros del consejo de administración 
- Exceso de códigos de conducta - Atresmedia, Servicios Informativos de Antena 3 y LaSexta - que induce a diferenciar los valores compartidos en la corporación

- Implantación de canales de interlocución con los grupos de interés, pero omisión total de las respuestas generadas

- Vaguedad en las cifras relativas a la plantilla. Los cambios de punto de vista en los indicadores proporcionados debilitan su credibilidad

- Prevalencia del discurso retórico en la descripción de la producción audiovisual generada, que tiende, además, a resaltar valores cuantitativos frente a cualitativos.

Por su parte, la memoria de RTVE presenta:

- Una fórmula de navegación que no permite el acceso aleatorio al contenido del informe, de especial relevancia, si tenemos en cuenta que su extensión ronda las 400 páginas

- Abundante material fotográfico de conjunto que promueve la identidad de la corporación como grupo

- Indicadores supeditados al cumplimiento de los Presupuestos Generales del Estado y al ahorro, aunque sobresale la ausencia de datos sobre las indemnizaciones por la asistencia a las sesiones del consejo de administración

- Mayor rigor en la información sobre la proporción de hombres y mujeres de la plantilla

- Información parcial de la respuesta generada por los grupos de interés, en particular la de los empleados y la de los representantes sindicales

Para finalizar, consideramos pertinente señalar que las memorias y los informes de responsabilidad social no tienen capacidad para cambiar los entornos económicos y sociales generales en los que operan las empresas. Como tampoco las compañías por si solas, ni la comunicación en particular. En momentos de crisis económica, es razonablemente comprensible que los grupos de interés concedan escasa o nula credibilidad a las memorias que no los tengan en cuenta como iguales u omitan datos que el sentido común puede situar fácilmente en un contexto económico desfavorable.

\section{Bibliografía}

AtRESMEDIA (2013): Informe Anual y de Responsabilidad Corporativa $2012 \mathrm{en}$ : http:// www.atresmediacorporacion.com/responsabilidad-corporativa/informes/ (Consulta $16 / 10 / 2014)$.

Barreda, J. (2006): «Propuestas del Foro de Expertos en RSE», Revista APD: Asociación para el Progreso de la Dirección, 212, 28-29.

BenAvides, J. (2008): «Los grupos de interés y la gestión de valores de responsabilidad social en las empresas y organizaciones», en BAJo, A. y Villagra, N. (ed.), Reflexiones en torno a la responsabilidad social en el ámbito de la globalización. Memoria académica curso 2007-2008. Madrid, Universidad Pontificia de Comillas, 99-114. 
Comisión Europea (2011): «Comunicación de la Comisión al Parlamento Europeo, al Consejo,alComitéEconómicoySocialEuropeoyalComitédelasRegiones», en:http:// eur-lex.europa.eu/LexUriServ/LexUriServ.do?uri=COM:2011:0681:FIN:es:PDF (Consulta 16/10/2014).

Corporación RTve (2014): Memoria de servicio público y Responsabilidad Social Corporativa 2012, en: http://www.rtve.es/corporacion/rc/ (Consulta 16/10/2014).

EDELMAN TRUST BAROMETER (2014): Resultados para España, en: http://www. edelmanspain.es/ (Consulta 16/10/2014).

García Perdiguero, T. (2003): La responsabilidad social de las empresas en un mundo global. Barcelona, Anagrama.

Global RePORTING InITIATIVE (GRI), en: https://www.globalreporting.org/languages/ spanish/Pages/default.aspx. (Consulta 16/10/2014).

Hermann, C. (2014): «El papel del sector público en el combate de la desigualdad», Boletín Internacional de Investigación Sindical, vol. 6, núm. 1, Ginebra, Oficina Internacional del Trabajo, 127-145. Disponible en: http://www.ilo.org/wcmsp5/ groups/public/---ed_dialogue/---actrav/documents/publication/wcms_247984.pdf (Consulta 16/10/2014).

Iso 26000 (2010): Guia de responsabilidad social, en: https://www.iso.org/obp/ ui/\#iso:std:iso:26000:ed-1:v1:es. (Consulta 16/10/2014).

López Triana, I. (2013): «Observatorio Global de Intangibles», en Villafañe, J. (dir.), La comunicación empresarial y la gestión de los intangibles en España y Latinoamérica. Informe anual 2013. Madrid, Pearson Educación, 3-24.

Mateos-Aparicio, P. (2007): Dirección y objetivos de la empresa actual. Madrid, Centro de Estudios Ramón Areces.

MíngUEZ, N. (2000): «Un marco conceptual para la imagen corporativa», ZER Revista de Estudios de Comunicación, 8, Bilbao, UPV/EHU, 321. Disponible en: http:// www.ehu.es/zer/es/hemeroteca/articulo/un-marco-conceptual-para-la-imagencorporativa/115 (Consulta 16/10/2014).

Polo, A. (2008): «El empresario ante la Ley moral», en BaJo, A. y Villagra, N. (ed.), Reflexiones en torno a la responsabilidad social en el ámbito de la globalización. Memoria académica curso 2007-2008. Madrid, Universidad Pontificia de Comillas, $67-74$.

Price Waterhouse Coopers (PwC) (2013): ¿Transmiten los informes el valor creado por las empresas? Análisis del estado del reporting integrado en las empresas del IBEX 35, en: http://www.pwc.es/es_ES/es/publicaciones/auditoria/assets/pwcreporting-integrado-2013.pdf (Consulta 16/10/2014).

SGE 21, en: http://foretica.org/index.php/presentacion?lang=es (Consulta 30/01/2015)

Social AcCountability InTERnATIONAL (SAI), en: http://www.sa-intl.org/index. $\mathrm{cfm}$ ?fuseaction=Page.ViewPage\&PageID=937 (Consulta 16/10/2014)

VV.AA. (2007): «Informe del Foro de Expertos en Responsabilidad Social de las empresas», Ministerio de Empleo y Seguridad Social, en: http://www.empleo. gob.es/es/sec_trabajo/autonomos/economia-soc/resposocempresas/foro_expertos/ index.htm (Consulta 16/10/2014)

Villafañe, J. (2004): La buena reputación. Claves del valor intangible de las empresas. Madrid, Pirámide. 
VILlagRA, N. (2008): «La RSC, su comunicación y la necesidad de una nueva conciencia», en Bajo, A. y Villagra, N. (ed.), Reflexiones en torno a la responsabilidad social en el ámbito de la globalización. Memoria académica curso 2007-2008. Madrid, Universidad Pontificia de Comillas, 127-142. 